\title{
Analytical evaluation of the roller bearing service life
}

\author{
Aleksey Antsupov ${ }^{1}$, Viktor Antsupov ${ }^{1}$, and Mikhail Slobodianskii ${ }^{1, *}$ \\ ${ }^{1}$ Department of Design engineering and operation of metallurgical machines and equipment, Nosov Magnitogorsk State Technical \\ University, 455000 Magnitogorsk city Lenin Street, 38 , Chelyabinsk Region, Russian Federation
}

\begin{abstract}
An analytical method has been developed for the design evaluation of the service life of roller bearings by the criterion of contact endurance of elements that does not require carrying out the experimental studies. It is based on the kinetic approach to the description of the solids destruction process and the theory of parametric reliability of machine parts. In contradistinction to the standard empirical dependence, which is currently used, the method allows one to take into account the effect of the material properties, and basic design and operational parameters on the service life. The results of the theoretical studies of the service life of roller bearing No. 212 (DIN 5412 T1) are presented for the quantitative assessment of this effect. They showed that the temperature-force loading conditions or material properties that were not taken into account might result in the prediction error of 1.5 or more times.
\end{abstract}

\section{Setting research objectives}

At present, a standard empirical dependence formulated based on the statistical analysis of bench test results is used in order to estimate the rated life $L_{10}$ (in hours) of roller bearings $(\mathrm{RB})[1,2]$ :

$$
L_{10}=\left(\frac{C}{P}\right)^{10 / 3} \cdot \frac{10^{6}}{60 \cdot n},
$$

where $C$ is a dynamic radial or axial load capacity of bearings, which is given in the standard [3] for each bearing size; $P$ is a dynamic equivalent radial or axial load, which is determined by the dependences [1]; $n$ is a constant rotation frequency of one of the bearing rings relative to another in rpm [2].

The standard [1] does not regulate the conditions of bench tests; it is only noted that a service life for bearings made of widely used material, being of a good quality, with a good quality of manufacture, and operating under normal operation conditions is determined by condition (1). It is obvious, that changes in the operation conditions comparing with the bench test conditions are not reflected in dependence (1). Additional bench tests and the introduction of new empirical coefficients into calculated dependence (1) are necessary to record such changes. For instance, in accordance with [1, 4], modification factors are introduced into equation (1) taking into account the effect on the design life of various reliability requirements, lubrication conditions, lubricant contamination, a fatigue load limit, oil film thickness, an axial load component and others. However, the introduction of additional coefficients reduces the accuracy of service life prediction and significantly increases material and time costs in stages of the bearing support development.

Thus, empirical dependence (1) does not describe physical regularities of the process of $\mathrm{RB}$ material structure damageability and does not allow one to predict the time of their failure with sufficient reliability because of surface contact fatigue failure. The issue on the theoretical evaluation of the RB design life without carrying out the experimental research remains open in contemporary literature despite the significant success of the machine parametric reliability theory $[5,6]$ and the kinetic concept of structural materials destruction $[7,8]$.

Therefore, the purpose of current research is the development of an analytical method for the design evaluation of the roller bearing service life by the criterion of the surface endurance of elements, as well as the carrying out the theoretical studies on the assessment of the effect of temperature-force operating conditions on their durability.

\section{The predicting method of the RB service life by the criterion of the fixed outer ring endurance}

The design life of the roller bearing under study is determined by the moment of occurrence of spalling of one of the rings or rolling bodies (rollers). To reduce the article length, we represent a method for predicting the average RB service life with a rotating inner ring by the contact strength criterion of a fixed outer ring. In addition, we consider temperature-force loading conditions stationary. The following groups of parameters are the initial data for the calculation.

The first group. The geometric and kinematic characteristics of the RB, the operation conditions: $d$,

\footnotetext{
* Corresponding author: m.slobodianskii@gmail.com
} 
$D, B$ are internal and external diameters of the $\mathrm{RB}$, its width; $D_{W}, l, z$ are roller diameter and length, their number; $n_{b}$ is the rotation frequency of the inner ring; $F_{r}=$ const is the radial load; $T_{0}=$ const is the ambient temperature; $T_{c M}=$ const is the steady operating temperature of the lubricant.

The second group. The physical and mechanical characteristics of the material of the outer ring of the RB: $E$ and $K$ are the modulus of linear and bulk elasticity; $G$ is the modulus of elasticity in shear; $\mu$ is Poisson's ratio; $\rho$ is the density; $V_{a m}$ is the atomic volume; $H V$ and $H B$ are Vickers and Brinell hardness; $\sigma_{B}$ is the strength limit; $\sigma_{T}$ is the yield point; $\sigma_{r}$ is the endurance limit.

The third group. The thermophysical characteristics of the material of the ring: $\Delta H_{T B}$ is the enthalpy of melting of the material in the solid state; $c$ is the specific heat capacity of the material; $\alpha_{0}$ is the coefficient of linear thermal expansion.

The fourth group. The physical constants: $k$ is the Boltzmann's constant; $h$ is the Planck's constant.

In accordance with the thermodynamic concept of predicting the reliability of machine parts $[6,9,10]$, the RB mean life under stationary load conditions can be determined by the equation:

$$
t_{50}=\left(u_{e^{*}}-u_{e 0}\right) / \dot{u}_{e},
$$

where $u_{e 0}$ is the latent energy density of the defects in the structure of the ring material in the initial state:

$$
u_{e 0}=\frac{\left((0,067 \cdot H V)^{1,2} \cdot k_{\sigma}\right)^{2}}{6 \cdot G} ;
$$

$k_{\sigma}$ is a complex structural parameter:

$$
k_{\sigma}=\left(6,47 \cdot 10^{-6} \cdot H V+0,12 \cdot 10^{-2}\right)^{-1} ;
$$

$u_{e^{*}}$ is the critical density of the latent energy of the material of the most loaded volume of the outer ring. The equation for its calculation is shown below.

The rate of damageability $\dot{u}_{e}$ of the structure material in condition (2) is determined from the kinetic equation of the form [9]:

$$
\begin{aligned}
& \dot{u}_{e}=k_{c m p} \cdot \frac{2 \cdot k \cdot T_{f}}{h} \cdot U\left(\sigma_{0}, T\right) \times \\
& \times \exp \left(-\frac{U\left(\sigma_{0}, T\right) \cdot V_{a m}}{k \cdot T_{f}}\right) \cdot \operatorname{sh}\left(\frac{A_{f}^{0} \cdot V_{a m}}{2 \cdot k \cdot T_{f}}\right) .
\end{aligned}
$$

We define the parameters entering into equation (4) in the sequence of their influence on each other $[9,10]$ :

The average temperature of self-heating of the deformable volume of the outer ring:

$$
T_{f}=T_{C M}+\frac{T_{*}-T_{C M}}{44 \cdot v_{0}}
$$

where $T_{*}$ is the local temperature in the contact zone:

$$
T_{*}=\frac{T_{c \mathcal{M}} \cdot\left(3 \cdot \sigma_{T}\right)^{2}}{\left(3 \cdot \sigma_{T}\right)^{2}-\sigma_{\max }^{2}} ;
$$

$\sigma_{\max }$ is the maximum stresses acting on the outer ring surface in the most loaded contact zone with the roller and determined from the reference dependencies [4] for each type of the RB;

$v_{0}=T_{0} / 870$ is the coefficient of uneven distribution of the internal energy density over the volume of the material.

The coefficient of resistance of the structure of the deformable volume of material in agreement with Le Chatelier's:

$$
k_{c m p}=\frac{2 \cdot\left(u_{e^{*}}-u_{e 0}\right)}{3 \cdot u_{e^{*}}-u_{e 0}},
$$

where $u_{e^{*}}$ is the critical density of the hidden energy:

$$
u_{e^{*}}=\Delta H_{T B}-c \cdot \rho \cdot T_{f} .
$$

The activation energy of the damage process of the material structure at temperature $T_{f}$ and stress $\sigma_{0}$ (taking into account the specific work of volume change $\left.A_{V}^{0}\right)$ :

$$
U\left(\sigma_{0}, T\right)=U\left(p_{T, 0}\right)-\Delta U_{T}-A_{V}^{0},
$$

where $U\left(p_{T 0}\right)$ is the initial activation energy taking into account the thermal pressure at $T_{0}$ and $\sigma_{0}=0$ determined from the empirical dependence of V.V. Fedorov [9, 10];

$\Delta U_{T}$ is a change in the activation energy from selfheating temperature $T_{f},{ }^{\circ} \mathrm{C}$ :

$$
\Delta U_{T}=1,5 \cdot \alpha_{0} \cdot K \cdot T_{f} ;
$$

$A_{V}^{0}$ is the specific energy of volume change under the conditions of the equivalent static load:

$$
A_{V}^{0}=\frac{M_{r}^{2} \cdot \varphi_{\sigma}^{2} \cdot \sigma_{0}^{2}}{2 \cdot K} ;
$$

$M_{r}$ is the coefficient of equivalence of the cyclic and static stresses:

$$
M_{r}=\left[\frac{\sigma_{T} \cdot(65+0,46 \cdot H V)}{\sigma_{r}^{2}}\right]^{0,5} ;
$$


$\varphi_{\sigma}$ is the coefficient of overvoltage on the interatomic bonds:

$$
\varphi_{\sigma}=k_{\sigma} \cdot v_{0}^{0,5}
$$

$\sigma_{0}$ is the average hydrostatic stress at the most dangerous point of the loaded volume under the working surface of the ring at the critical depth $[9,11]$ :

$$
\sigma_{0}=\frac{\sigma_{1}+\sigma_{2}+\sigma_{3}}{3}
$$

$\sigma_{1} ; \sigma_{2} ; \sigma_{3}$ are the main stresses at the dangerous point: $\quad \sigma_{1}=-0,18 \cdot \sigma_{\max } ; \quad \sigma_{2}=-0,976 \cdot \sigma_{\max }$; $\sigma_{3}=-0,78 \cdot \sigma_{\max }$.

Specific energy of forming under conditions of the equivalent static load:

$$
A_{f}^{0}=\frac{M_{r}^{2} \cdot \varphi_{\sigma}^{2} \cdot \sigma_{i}^{2}}{6 \cdot G},
$$

where $\sigma_{i}$ is the intensity of normal stresses at the most dangerous point under the working surface of the ring $[9,11]$ :

$$
\sigma_{i}=\sqrt{\frac{\left(\sigma_{1}-\sigma_{2}\right)^{2}+\left(\sigma_{2}-\sigma_{3}\right)^{2}+\left(\sigma_{1}-\sigma_{3}\right)^{2}}{2}} .
$$

After the average design life $t_{50}$ had been found by condition (2), a ninety percentile life $t_{90}$ was estimated under the assumption of a normal distribution of the time to failure $t_{*}$ as a random variable (quantile $u_{90}=-1,282$ ) with the value of the coefficient of variation $v_{t}=0,125$ :

$$
t_{90}=t_{50}+u_{90} \cdot v_{t} \cdot t_{50} \approx 0,84 \cdot t_{50} .
$$

An algorithm and the program "Durability of the RB", which makes it possible to estimate $t_{50}$ and $t_{90}$ under given stationary load conditions, were created based on the proposed method (2) - (17).

The theoretical studies have been carried out with the use of this program to assess the effect of temperatureforce operating conditions on the roller bearing life.

\section{Analysis of computer experiment results}

We carried out the theoretical studies for single-row roller bearing no. 212 (DIN 5412 T1) with short cylindrical rollers. In the example given in the study [4], its nominal life calculated from the standard empirical dependence (1) with the radial load $F_{r}=5600 H$ and the rotational speed of the inner ring $n_{b}=800 \mathrm{rpm}$ was $L_{10}=41757 \mathrm{~h}$.

The initial groups of bearing parameters were matched such that the theoretical life $t_{90}$ at $F_{r}=5600 \mathrm{H}$ and $n_{B}=800 \mathrm{rpm}$ was approximately equal to the empirical one $L_{10}=41757 h$ to ensure that the results of studies on the life time of RB no. 212 on empirical relationship (1) and theoretical method (2) - (17) were comparable. RB no. 212 manufactured, for instance, from SHX15 with hardness $H B \approx 5820 \mathrm{MPa}$ and yield strength $\sigma_{T} \approx 2000 \mathrm{MPa}$ corresponds to this condition. In this case, the stable operating temperature of the lubricant should be equal to $T_{c M} \approx 80^{\circ} \mathrm{C}$. Then, its theoretical design life is $t_{90}=41743 h$ and is approximately equal to $L_{10}=41757 \mathrm{~h}$. The graphical interpretation of these solutions is shown in Figure 1 in the direction of the $a-b$ pointers.

This figure also shows the change in the empirical life $L_{10}$ (curve ISO 281:2007) and the theoretical life $t_{90}$ (curves 1-9) of $\mathrm{RB}$ no. 212 when changing the operating conditions: $F_{r}=1000 \div 8000 H \quad$ and $T_{c M}=20 \div 100^{\circ} \mathrm{C}$.

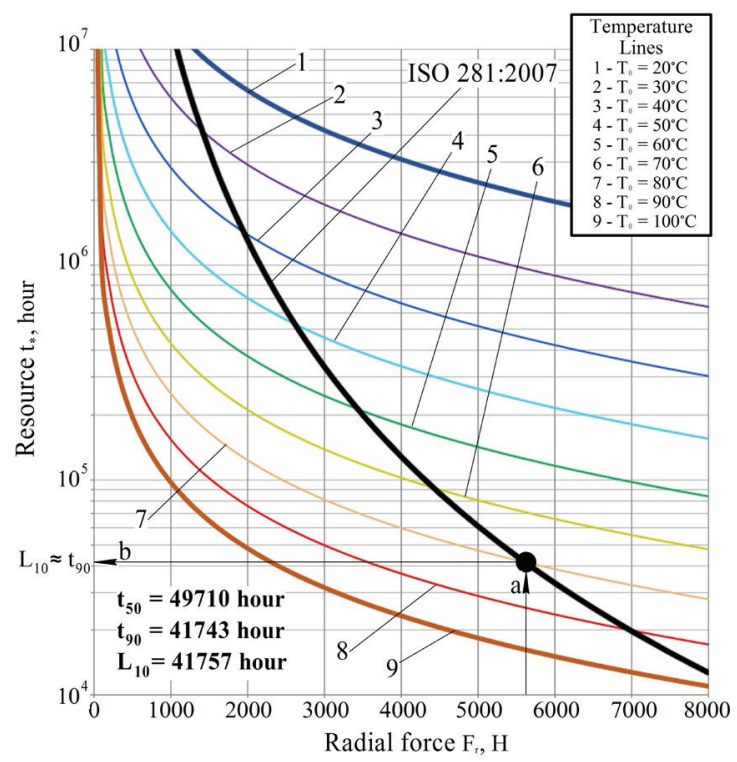

Fig. 1. The effect of temperature-power parameters on the service life of bearing no. 212 (DIN 5412 T1).

The results show the following:

- the operating temperature is the most important parameter, which the empirical dependence does not explicitly take into account (1). The figure shows that, in the example considered, a change in the temperature in the range $T_{c M}=20 \div 100^{\circ} \mathrm{C}$ at constant load $F_{r}=5600 H$ can result in a change in the life time by 2 orders of magnitude;

- the change in the radial load in the specified range results in a change in the life by 3 orders of magnitude (from $\sim 10^{7}$ hours to $\sim 10^{4}$ hours ). At the same time, the corresponding change in the theoretical life is only one order (from $\sim 10^{4}$ hours to $\sim 10^{3}$ hours ). A more abrupt decline in the empirical curve is evidently because dependence (1), simultaneously with the increase of the load $F_{r}$, implicitly takes into account the natural 
increase in the operating temperature of the bearing lubricant in bench tests. The nomogram shows that the operating temperature should increase from $T_{c M} \approx 20^{\circ} \mathrm{C}$ to $T_{c M} \approx 100^{\circ} \mathrm{C}$. The theoretical curve is created for one constant value $T_{C_{M}} \approx 80^{\circ} \mathrm{C}$.

It is obvious that the proposed method allows one to investigate the effect of other initial parameters on the value of the project life of the RB under study without carrying out additional experiments. For instance, calculations show that a change in the hardness of bearing steels in the range $H B \approx 5820 \div 6700 \mathrm{MPa}$ [4] under other equal conditions of the example under consideration results in the change in the life time of no less than 1.5-1.7 times. The empirical curve also does not consider this.

The possibilities described favourably distinguish the proposed analytical approach from the unambiguous experimental dependence for estimation of the roller bearing service life by the dynamic load capacity.

\section{Conclusion}

1. It is noted that now at the design stage of bearing supports their life is determined by the standard empirical dependence that was obtained by the results of bench tests. The change in operating conditions requires additional experimental studies that significantly increases the cost and lengthens the development stages. 2. An analytical technique for predicting the life of roller bearings by the energy criterion of contact endurance is formulated based on the modern kinetic approach to the destruction of solids and the thermodynamic theory of strength of structural materials. It allows one to take into account the effect of the properties of RB materials, the main design, and operational parameters on the durability without additional experiments.

3. The theoretical studies carried out showed that taking into account changes in the properties of materials or temperature-force operating conditions can result in a significant change in the service life no less than 1.5 and more times.

\section{References}

1. ISO 281:2007 Roller bearings. Dynamic load rating and basic rated life

2. M.N. Ivanov, V.A. Finogenov, Machine parts: Textbook for machine-building specialties of high schools (Higher Education, Moscow, 2008)

3. GOST 3395-89 Roller bearings. Types and constructive performances

4. L.Ya. Perel, A.A. Filatov, Rolling bearings: Calculation, design and maintenance of supports: Handbook (Mechanical Engineering, Moscow, 1992)

5. A.S. Pronikov, Machine Parametric Reliability (N.E. Bauman MSTU Publ., Moscow, 2002)
6. A.V. (Jr.) Antsupov, A.V. Antsupov, V.P. Antsupov Theory and practice of ensuring the reliability of machine parts by the criteria of kinetic strength and wear resistance of materials (Publishing house NMSTU, Magnitogorsk, 2015)

7. V.R. Regel, A.I. Slutsker, E.E. Tomashevskyi, Kinetic character of strength of solid bodies (Chief editorial board of physical and mathematical literature of the publishing center «Science», 1974)

8. G.P. Cherepanov, Strength problems 11, pp.3-8. (1989)

9. A.V. Antsupov, A.V. Antsupov(Jr.), V.P. Antsupov Procedia Engineering 150, p.726-733 (2016).

10. V.V. Fedorov, Basics of ergodynamics and synergy of deformable bodies (Izd HPE "KSTU", Kaliningrad, 2014)

11. K.V. (Ed.) Frolov, Modern tribology: Results and perspectives (Publishing House of LCI, Moscow, 2008) 